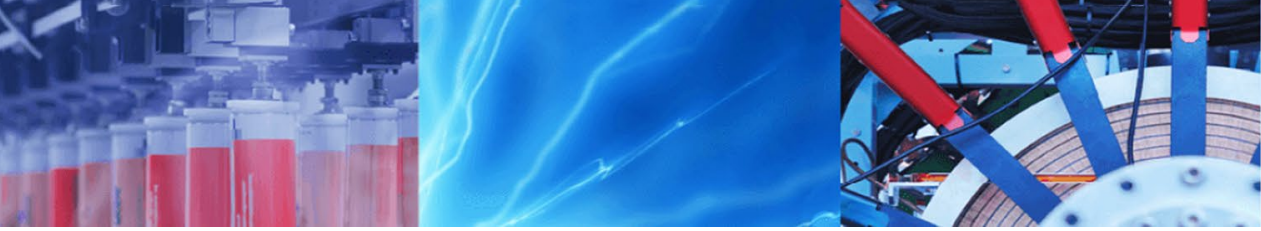

Research Article

\title{
Dielectric resonator loaded high-gain half-mode substrate integrated waveguide triangular antenna for microwave imaging and radar applications
}

\author{
Soumen Banerjee ${ }^{1}$ (1) . Susanta Kumar Parui ${ }^{1} \cdot$ Biswarup Rana $^{2}$
}

(c) Springer Nature Switzerland AG 2019

\begin{abstract}
In this paper, an equilateral triangular HMSIW antenna excited with coaxial feed is chosen at $5.28 \mathrm{GHz}_{\mathrm{H}}$ representing $\mathrm{TE}_{101}$ mode with next higher $\mathrm{TE}_{202}$ mode operating at $9.57 \mathrm{GHz}$ frequency in the $\mathrm{X}$-band. Two sides of the antenna with fitted with metallic vias while the third side remains barren. The antenna is found to exhibit gain of $5.7 \mathrm{dBi}$ and $7.5 \mathrm{dBi}$ for the $\mathrm{TE}_{101}$ and $\mathrm{TE}_{202}$ mode respectively. A rectangular box-shaped dielectric resonator (DR) is now placed over the radiating wall. The antenna-DR composite structure resonating at the higher $\mathrm{TE}_{202}$ mode at $9.5 \mathrm{GHz}$ and depicting an enhanced gain of $12 \mathrm{dBi}$ is presented here for X-band microwave imaging and radar applications. All antennas are made-up with Arlon AD270 substrate and simulation results show a close resemblance when compared with measured ones.
\end{abstract}

Keywords Antenna gain · Substrate integrated waveguide (SIW) - Dielectric resonator (DR) · Ground-penetrating radar $(\mathrm{GPR}) \cdot$ Microwave imaging $\cdot$ Synthetic aperture radar (SAR)

\section{Introduction}

The successful development of commercial RF broadband system depends on cost effectiveness, mass-producibility, superior-performance ability and high quality microwave and millimetre wave technologies. At millimetre wave, the circuit building blocks and antennas are very closely related. Though classical metallic waveguide still remains as the mainstream for designing superior-performance millimetre wave systems, it is not suitable for en-mass production with lower cost. In addition to its heavy weight and volume, the costly and cumbersome process of its tuning post-fabrication along with difficulty in assembling makes it unsuitable for widespread applications and use. Moreover other challenging problems involve designing of low loss ICs. Tremendous research worldwide is being carried out to develop high-performance microwave and millimetre wave components that can be fabricated using low cost technologies. A promising candidate for alleviating all such problems is the development of substrate integrated waveguide (SIW) technology [1-3]. This structure exhibits the propagation characteristics of classical waveguide including field pattern and dispersion characteristics as well as preserves all the advantages of the latter. By this technology the non-planar waveguides are converted into planar form structure which are compatible with printed circuit board (PCB) technology or other prevalent planar processing techniques like low-temperature co-fired ceramic (LTCC) technology.

Microwave imaging, an important and essential tool, has been developed to analyze embedded objects in a medium. It offers a plethora of applications in a variety of areas including medical imaging, concealed object detection for security, structural engineering, through-the-wall imaging, etc. [4]. However, security and medical imaging received significant importance in the last decade.

\footnotetext{
$\varangle$ Soumen Banerjee, prof.sbanerjee@gmail.com | ${ }^{1}$ Department of Electronics and Telecommunication Engineering, Indian Institute of Engineering Science and Technology (IIEST), Shibpur, Howrah 711103, India. ${ }^{2}$ Department of Electrical and Information Engineering, Seoul National University of Science and Technology, Seoul 139-743, South Korea.
}

SN Applied Sciences (2019) 1:811 | https://doi.org/10.1007/s42452-019-0837-1

Received: 18 February 2019 / Accepted: 25 June 2019 / Published online: 1 July 2019 
The non-invasive profile of microwave imaging helped in promising detection of breast cancer at an early stage [5]. Tomography coupled microwave systems have been reported to be successful in accurately extracting malignant tumor information and reconstruction [6]. In [7], the authors have further proposed a new low cost technique to construct 3D images for medical and security applications using indirect holographic approach along with microwave imaging. Consequently, efficient microwave imaging technique development is of utmost importance in medical, structural and security applications. The synthetic aperture radar (SAR) used to create 2 or 3-D images of objects or the ground-penetrating radar (GPR) used for geological observations are very popular qualitative microwave imaging methods. They calculate qualitative profile to represent hidden objects. It is herein that the demand of developing antennas as microwave sensing element has essentially attracted the attention of researchers worldwide. In this context, half-mode substrate integrated waveguide (HMSIW) based sensing antenna elements have been developed to work at both S- and C-band [8]. Also a CP DRA array with enhanced gain has recently been developed for microwave imaging applications [9].

The SIW based components and antennas marks a revolutionary change in construction of all electrical microwave and millimetre wave electronics component structures. They eliminate cross talks in sensitive microwave and millimetre wave circuits owing to their high EMI isolation property; provide enhanced signalling performance due to low conductor loss and provide excellent frequency stability for coupled filters and resonators owing to the capability of able to handle more power with proper electrical shielding. The SIW technology offers assimilation of passive and active elements including antennas on the alike substrate which creates system-in-package (SiP) with additional extension to system-on-substrate (SoS) enabling mounting of a single or multiple chip sets on a single substrate. In the last decade, tremendous growth and research has been witnessed in developing SIW based antennas for communication systems. Several antenna structures have already been proposed that are available in literature which includes the classical slotted-waveguide antennas, leaky-wave antennas, modified Vivaldi radiator, cavity-backed SIW antennas, H-plane sectoral horn antenna, HMSIW antennas, quarter-mode SIW (QMSIW) antennas, eighth-mode SIW (EMSIW) antennas, etc. The HMSIW [10-16], a special structure leading to miniaturization of antenna footprint, results from the division of fullmode SIW (FMSIW) along the quasi-magnetic wall keeping the field circulation identical to that of FMSIW structure.

The dielectric resonators (DRs), on the other hand, fabricated using low loss materials with high permittivity are very popular and are extensively used worldwide in microwave filter circuits and in other microwave structures like oscillators. They generally possess very high unloaded Q-factor with exception in open environment where reduction of $Q$ value for the lowest order modes are observed; a property depicting their suitability in use as antenna. The DR antennas (DRAs) have emerged as promising candidate for microwave and millimetre wave communication systems owing to their several eye-catching features like small size, low cost, greater impedance bandwidth, more radiation efficiency and tolerance to temperature variation, versatility, flexibility and ease in excitation techniques [17-19]. The DRAs offers the choice of excitation through various methods like feeding through microstrip line, conformal strip, coaxial probe, aperture coupling by microstrip line or coplanar waveguide, etc. and can be designed using various shapes like rectangle, cylinder, hemispherical, spherical and others.

In this paper, a HMSIW equilateral triangular antenna is designed by authors [16] with metallic vias array placed along two sides constituting perfect electrical conducting (PEC) wall with the other wall as perfect magnetic conducting (PMC) being empty of any such vias. Coaxial feeding technique is used offering ease in fabrication, easy impedance matching and at the same time generating low spurious radiation. The resonating frequency of the antenna for $\mathrm{TE}_{101}$ fundamental mode occurs at $5.28 \mathrm{GHz}$ generating $5.7 \mathrm{dBi}$ gain and upon varying the feed position the next higher $\mathrm{TE}_{202}$ mode at $9.57 \mathrm{GHz}$ is excited with a gain of $7.5 \mathrm{dBi}$. A rectangular box shaped DR chosen and placed on top of the PMC wall of the HMSIW triangular antenna. The antenna loaded with DR is found to be radiating at the higher $\mathrm{TE}_{202}$ mode of $9.57 \mathrm{GHz}$. The radiation from the PMC edge excites the DR and the antenna is found to exhibit a gain of $12 \mathrm{dBi}$ which is an excess gain of $4.5 \mathrm{dBi}$. All simulation studies are carried out using ANSYS make HFSS v15.0 and such studies are being tallied with experimentally measured ones.

\section{HMSIW based triangular antenna with rectangular box-shaped DR}

\subsection{The antenna configuration}

The geometrical structure of the rectangular box-shaped DR loaded parent antenna [16] excited through coaxial feeding technique is shown in Fig. 1. For the antenna, the length of the sides $(\mathrm{S})=31.18 \mathrm{~mm}$ with boundaries being $60(\mathrm{~L}) \times 60$ (W) $\times 0.79 \mathrm{~mm}^{3}$. It is designed using Arlon AD270 substrate (tm) (dielectric constant, $\varepsilon_{\mathrm{r}}=2.7$, thickness $\mathrm{h}=0.79 \mathrm{~mm}$, and loss tangent, $\tan \delta=0.002$ ). The metallic vias are positioned along the two edges to resemble PEC walls while the other one with no vias resemble PMC wall. The vias' diameter is 


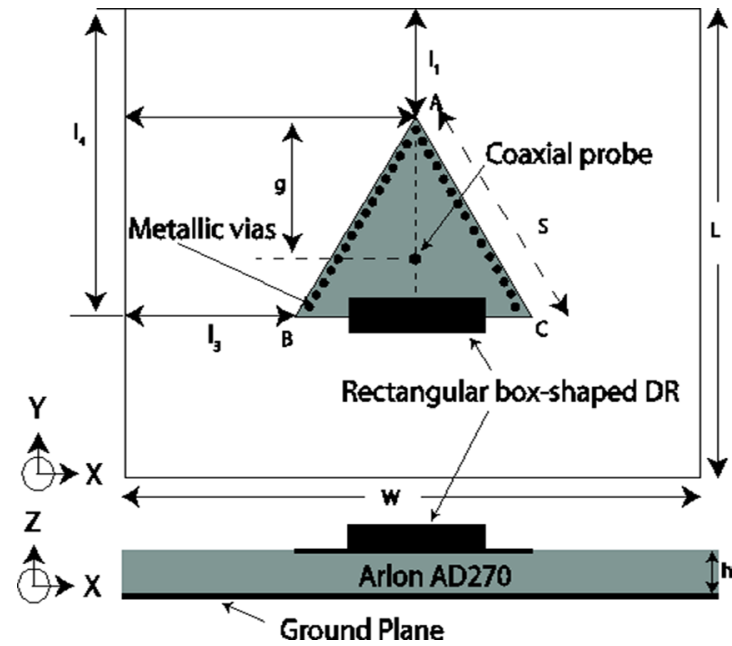

Fig. 1 Geometrical structure with necessary dimensions of the HMSIW equilateral triangular antenna loaded with rectangular box-shaped DR. Antenna dimensions: $\mathrm{L}=60 \mathrm{~mm}$,

chosen as $1 \mathrm{~mm}$ with distance between two centres $=1.5 \mathrm{~mm}$. Vias placed at $0.7 \mathrm{~mm}$ apart from the edges of the triangle curtails the actual radiation area as compared to triangular patch's physical area. The $\mathrm{TE}_{101}$ fundamental mode is excited through coax feeding at optimized position $(\mathrm{g})=14 \mathrm{~mm}$ while for $\mathrm{TE}_{202}$ mode, the next higher mode, excitation occurs at $\mathrm{g}=22 \mathrm{~mm}$. Thus proposed SIW based antenna is approximated through use of formulae for that of HMSIW antenna $[10,20]$ whose equivalent length and width are given by

$W_{\text {eff }}^{\text {HMSIW }}=\frac{W_{\text {eff }}^{\text {SIW }}}{2}+W$

$L_{\text {eff }}^{H M S I W}=\frac{L_{\text {eff }}^{S I W}}{2}+W$

where $L_{\text {eff }}^{S I W}$ and $W_{\text {eff }}^{S I W}$ are the SIW resonator's equivalent length and width. The use of non-linear least squares method facilitates the calculation of additional width $W[10,21]$

$$
\begin{aligned}
W= & h\left(0.05+\frac{0.3}{r}\right) \\
& \times \ln \left(0.79 \frac{W_{\text {eff }}^{S I W}}{4 h^{3}}+\frac{52 W_{\text {eff }}^{S I W}-261}{h^{2}}+\frac{38}{h}+2.77\right)
\end{aligned}
$$

For the $\mathrm{TE}_{\mathrm{m} 0 \mathrm{p}}$ modes of the HMSIW triangular antennas, the resonant frequency calculation is through the use of equations related to SIW counterpart structure [22]

$$
f_{m n p}^{H M S I W}=\frac{1}{2 \pi \sqrt{\mu}} \sqrt{\left(\frac{m \pi}{L_{e f f}^{H M S I W}}\right)^{2}+\left(\frac{p \pi}{W_{e f f}^{H M S I W}}\right)^{2}}
$$

Antenna dimensions:

$\mathrm{L}=60 \mathrm{~mm}, \mathrm{~W}=60 \mathrm{~mm}, \mathrm{~S}=31.18 \mathrm{~mm}, \mathrm{~h}=0.79$ $\mathrm{mm}, \mathrm{l}_{1}=12 \mathrm{~mm}, \mathrm{l}_{2}=30 \mathrm{~mm}, \mathrm{l}_{3}=14.41 \mathrm{~mm}, \mathrm{l}_{4}=$ $39.01 \mathrm{~mm}$

DR dimensions:

Length $=19.05 \mathrm{~mm}$, Breadth $=9.525 \mathrm{~mm}$, Height $=$ $4.7 \mathrm{~mm}$.
$\mathrm{W}=60 \mathrm{~mm}, \mathrm{~S}=31.18 \mathrm{~mm}, \mathrm{~h}=0.79 \mathrm{~mm}, \mathrm{I}_{1}=12 \mathrm{~mm}, \mathrm{I}_{2}=30 \mathrm{~mm}$, $\mathrm{I}_{3}=14.41 \mathrm{~mm}, \mathrm{I}_{4}=39.01 \mathrm{~mm}$. DR dimensions: Length $=19.05 \mathrm{~mm}$, Breadth $=9.525 \mathrm{~mm}$, Height $=4.7 \mathrm{~mm}$

A rectangular box-shaped isolated $D R$ is chosen and placed over the SIW antenna as shown in Fig. 1. The DR with rectangular cross-section is the most versatile one owing to its flexibility arising out of two degrees of freedom; allowing wide range of aspect ratios for a given design frequency. The rectangular DR offers hindrance in analysis for increase in edge-shaped boundaries. However, its choice over cylindrical and spherical DR is owing to the fact that the resonating frequencies of the various excited modes can be made to differ from each other through proper choice of the three dimensions of the resonator. Further, compact DRA with small footprint can be achieved, or very low profile is realizable, or adjustment in its bandwidth is achievable for a given material permittivity. The DRA's operating band width can be easily controlled and tuned over a larger range through suitable choice of resonating parameters. For lower order modes of DR antenna, the bandwidth can be varied from a fraction of a percent to about $10 \%$ or more through variation in dielectric constant of the resonator material. Another advantage over this flexibility is that various types of feeding mechanisms can be used to excite the rectangular DRA, thereby making it responsive to integration with current available technology. A fairly simple use of dielectric waveguide model enables fairly accurate prediction of resonant frequency and bandwidth of the rectangular DRA rather than resorting to time-consuming numerical methods.

The rectangular box-shaped DR of length $=19.05 \mathrm{~mm}$, breadth $=9.525 \mathrm{~mm}$, height $=4.7 \mathrm{~mm}$ and of dielectric constant 20 is directly placed partly on top of a metallic plane and partly on top of a low permittivity dielectric Arlon AD270 with dielectric constant 2.7. This results in 


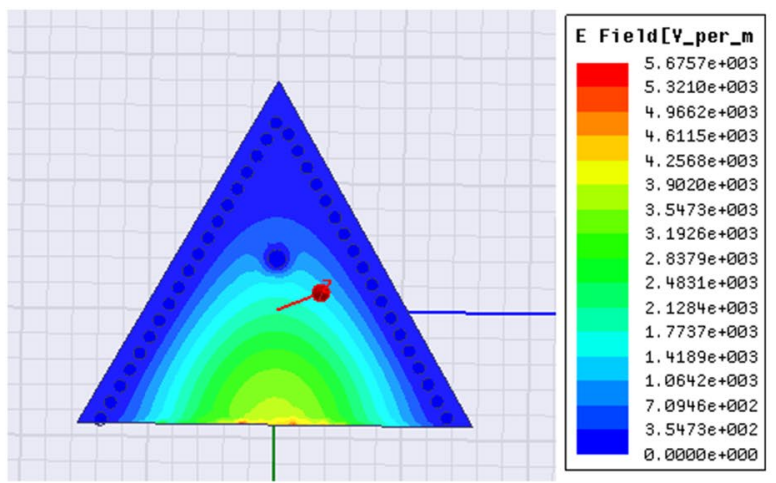

(a)

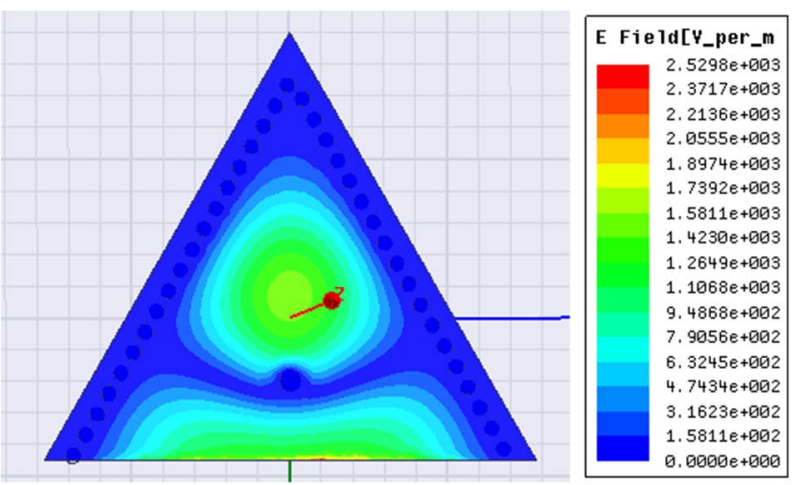

(b)

Fig. 2 Pattern of distribution of Electric field for HMSIW equilateral triangular antenna without rectangular box-shaped DR a TE 101 fundamental mode $(5.28 \mathrm{GHz}), \mathbf{b ~ T E}_{202}$ higher mode $(9.57 \mathrm{GHz})$

enhancement of bandwidth of each resonance mode owing to the lowering of Q-factor value. The authors have used here Emerson and Cuming make Eccostock HIK DR and its dimensions are optimized for best antenna performance.

\subsection{Simulated and experimental results}

The location of the feed position for the HMSIW equilateral triangular antenna for both the $\mathrm{TE}_{101}$ and $\mathrm{TE}_{202}$ modes are carried out through parametric studies. The electric field distribution for the parent HMSIW equilateral triangular antenna for the two modes is depicted in Fig. 2 while the same for the antenna-DR composite structure is shown in Fig. 3. Owing to DR loading, the effective permittivity of the antenna-DR composite structure increases resulting in excitation of higher order mode. Thus Fig. 3 clearly revels that the higher order $\mathrm{TE}_{202}$ mode is excited with the inclusion of DR. The separation of high-permittivity DR from the ground plane by the low-permittivity dielectric substrate forces all the electric field lines to circulate through the low-permittivity region. The prototypes of the antennas fabricated using Arlon substrate is depicted in Fig. 4. From the parametric studies of feed location for excitation of modes in a HMSIW triangular antenna, it is clearly evident from Fig. $5 \mathrm{a}$ that for $\mathrm{TE}_{101}$ mode the feed location is at $\mathrm{g}=14 \mathrm{~mm}$ while the same for higher order $\mathrm{TE}_{202}$ mode is at $\mathrm{g}=22 \mathrm{~mm}$ as shown in Fig. $5 \mathrm{~b}$. In case of $\mathrm{TE}_{101}$ mode, the final simulated return loss observed through HFSS is $-29 \mathrm{~dB}$ at $5.28 \mathrm{GHz}$ while the same experimentally obtained from Agilent VNA is found to be $-26 \mathrm{~dB}$ at $5.34 \mathrm{GHz}$ as depicted in Fig. $6 \mathrm{a}$. Also for $\mathrm{TE}_{202}$ mode, the optimized simulated and experimental $\mathrm{S}_{11}$, as shown in Fig. $6 \mathrm{~b}$, is $-17 \mathrm{~dB}$ at $9.57 \mathrm{GHz}$ and $-28 \mathrm{~dB}$ at $9.61 \mathrm{GHz}$ respectively. The span of $5.25-5.30 \mathrm{GHz}$ impedance bandwidth, i.e., $0.95 \%$ fractional bandwidth is achieved for $\mathrm{TE}_{101}$

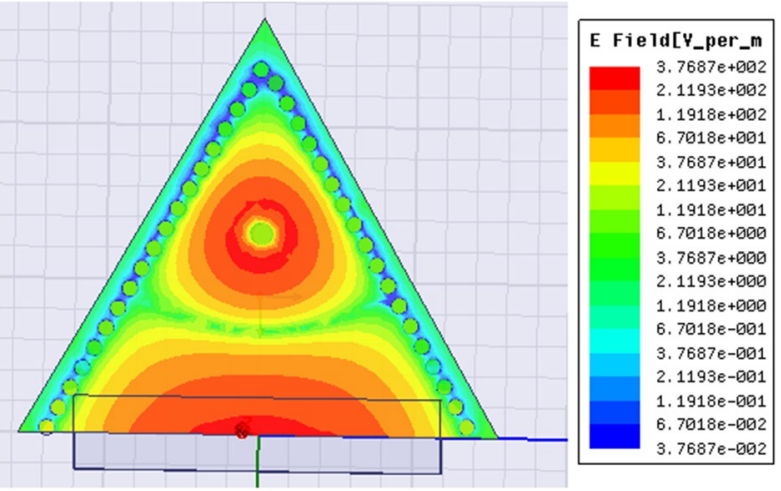

Fig. 3 Electric field distribution for the antenna with DR loading: $\mathrm{TE}_{202}$ mode $(9.5 \mathrm{GHz})$

mode while a $60 \mathrm{MHz}$ impedance bandwidth covering 9.53-9.59 GHz is obtained for $\mathrm{TE}_{202}$ mode.

The antenna when supported with a rectangular boxshaped DR over the PMC wall, exhibits a simulated reflection coefficient value of $S_{11}=-40 \mathrm{~dB}$ at $9.5 \mathrm{GHz}$ which is obtained through parametric studies upon variation of $D R$ dimensions as shown in Fig. 7. A plot of $S_{11}$ against frequency upon variation in length of DR is plotted in Fig. 7a while variation in return loss upon individual changes in DR breadth and height is carried out as shown in Fig. 7b, c respectively. Thus the optimized DR dimensions obtained are 19.05 (length LD) $\times 9.525$ (breadth BD) $\times 4.7$ (height $\mathrm{HD}) \mathrm{mm}^{3}$. The experimentally obtained return loss of the antenna-DR composite structure is $-35 \mathrm{~dB}$ at $9.7 \mathrm{GHz}$ and a comparison of it with simulated one is plotted in Fig. 7d. From the figure, both simulated and experimentally obtained values are nearly close to each other, however, a slight variation in the two values occurs owing to the several fabrication defects associated with the antenna and the mismatch occurring in the circuit with the SMA 
Fig. 4 Fabricated prototype of the HMSIW equilateral triangular antennas a without DR, b with rectangular box-shaped DR

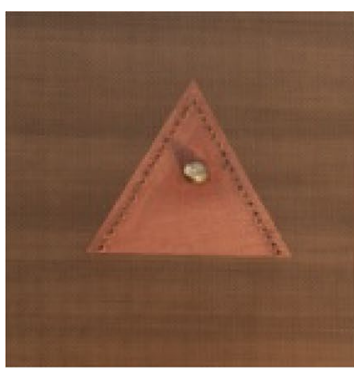

(a)

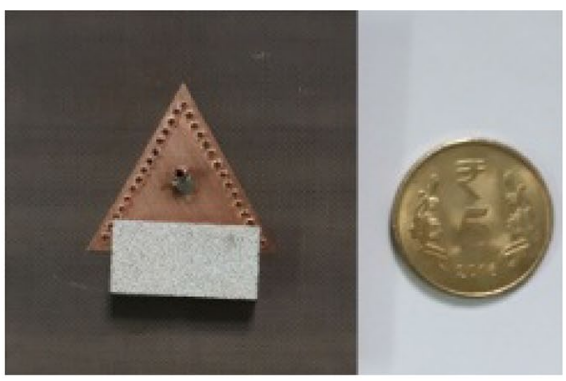

(b)

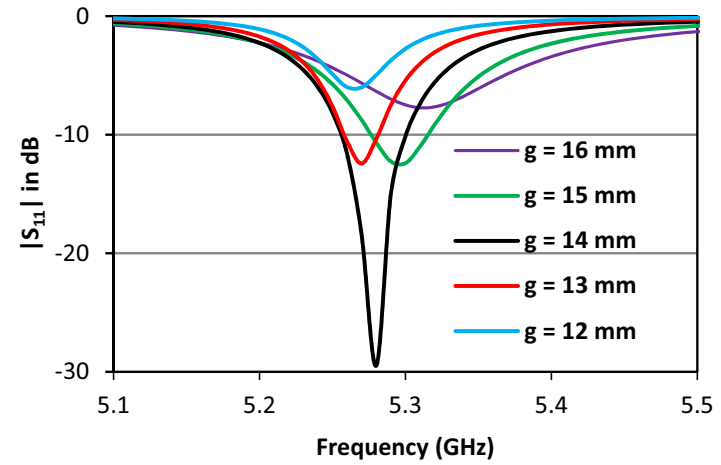

(a)

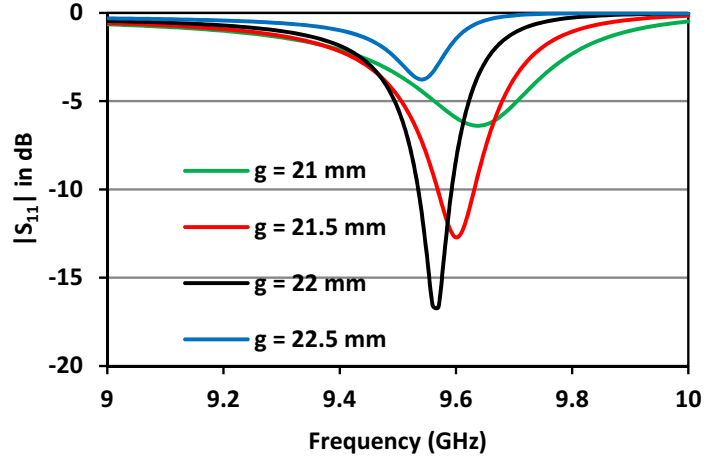

(b)

Fig. 5 Return loss variation versus feed point location of the HMSIW triangular antenna for $\mathbf{a} \mathrm{TE}_{101}$ mode and $\mathbf{b} \mathrm{TE}_{202}$ mode

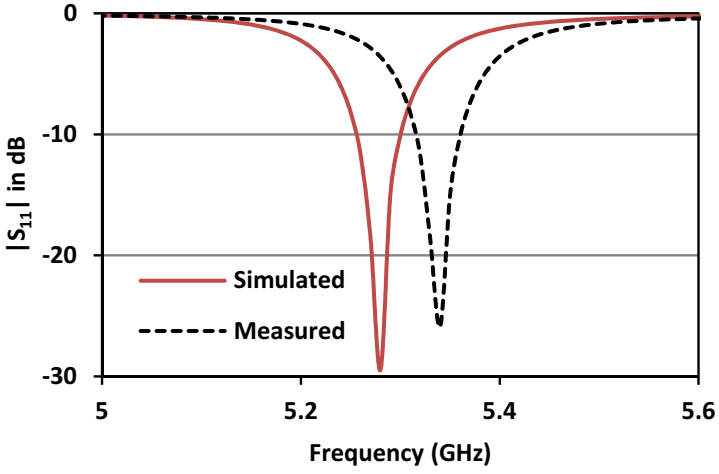

(a)

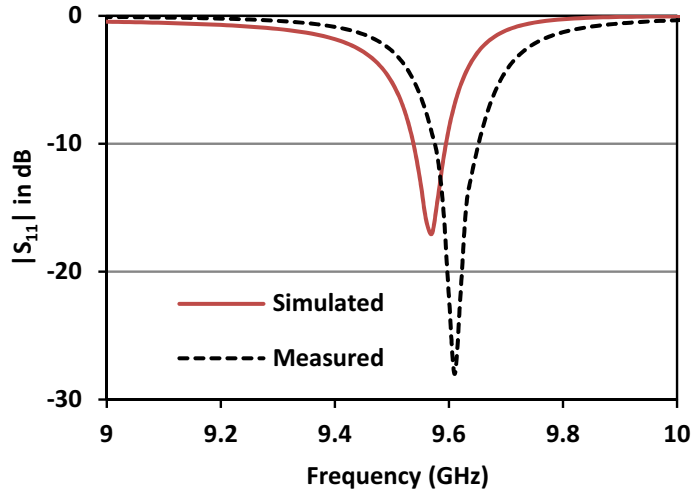

(b)

Fig. 6 Variation of $S_{11}$ with frequency of the antenna for $\mathbf{a} T E_{101}$ mode and $\mathbf{b} T E_{202}$ mode

connector. The impedance bandwidth for the composite structure is found to be $420 \mathrm{MHz}$ spanning from 9.28 to $9.70 \mathrm{GHz}$, i.e., a fractional bandwidth of $4.4 \%$.

From the simulated plot in Fig. $7 \mathrm{~d}$, it is observed that after loading with $\mathrm{DR}$, two more close resonances beside the desired resonance at $9.5 \mathrm{GHz}\left(\mathrm{TE}_{202}\right.$ mode) are seenthe first one occurring at $9.34 \mathrm{GHz}$ owing to resonance of the rectangular box-shaped $\mathrm{DR}$ exciting the $\mathrm{TE}_{211}$ mode and the other at $9.68 \mathrm{GHz}$ owing to higher order $\mathrm{TE}_{303}$ mode excitation of the SIW antenna itself resulting in a combined effect of wider bandwidth for the Antenna-DR composite structure. The corresponding electric field patterns for the DR ( $\mathrm{TE}_{211}$ mode) and the SIW antenna $\left(\mathrm{TE}_{303}\right.$ mode) are depicted in Fig. 8a, b respectively.

The variation of gain of the HMSIW triangular antenna is revealed in Fig. 9 for the $\mathrm{TE}_{101}$ and $\mathrm{TE}_{202}$ modes at 


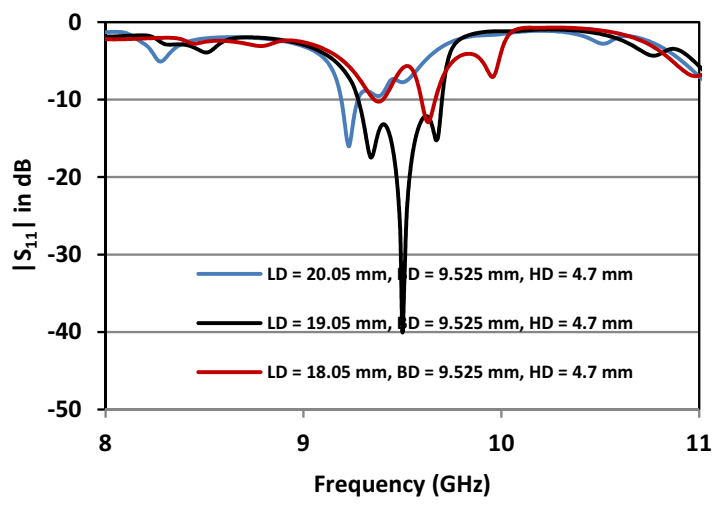

(a)

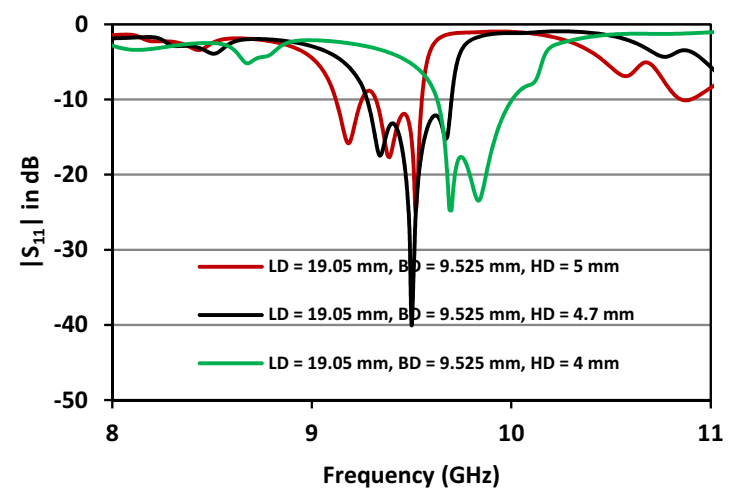

(c)

Fig. 7 Variation of $\mathrm{S}_{11}$ with dimension of the rectangular boxshaped DR in the HMSIW triangular antenna for $\mathrm{TE}_{202}$ mode $(9.5 \mathrm{GHz})$ - $\mathbf{a}$ variation in length (LD) of $\mathrm{DR}$, $\mathbf{b}$ variation in breadth (BD) of DR, c variation in height (HD) of DR and, $\mathbf{d}$ both simulated

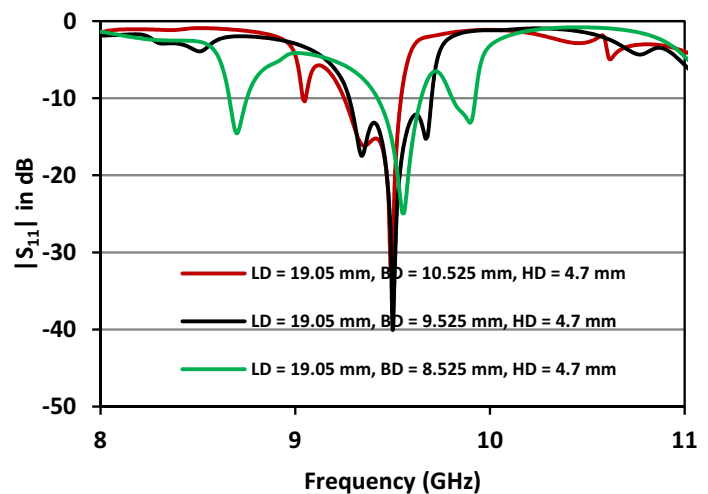

(b)

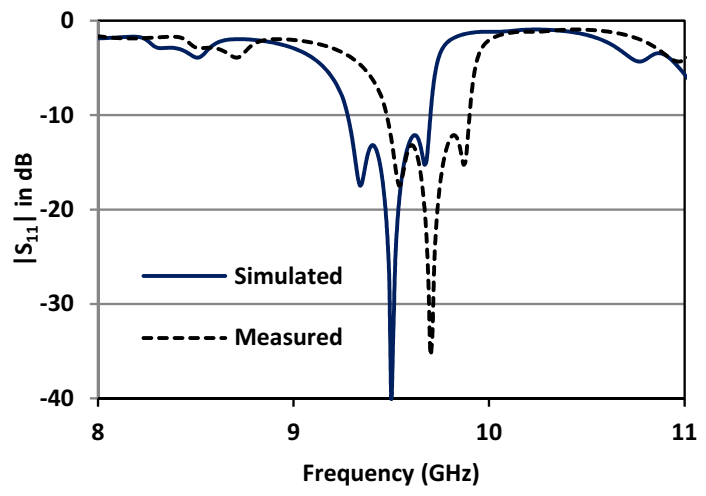

(d)

and measured $S_{11}$ versus frequency for optimized DR dimensionslength $(L D)=19.05 \mathrm{~mm}$, breadth $(B D)=9.525 \mathrm{~mm}$ and height $(\mathrm{HD})=4.7 \mathrm{~mm}$
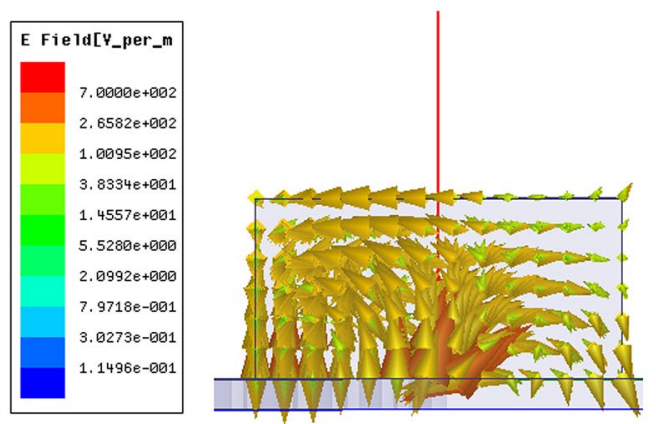

(a)
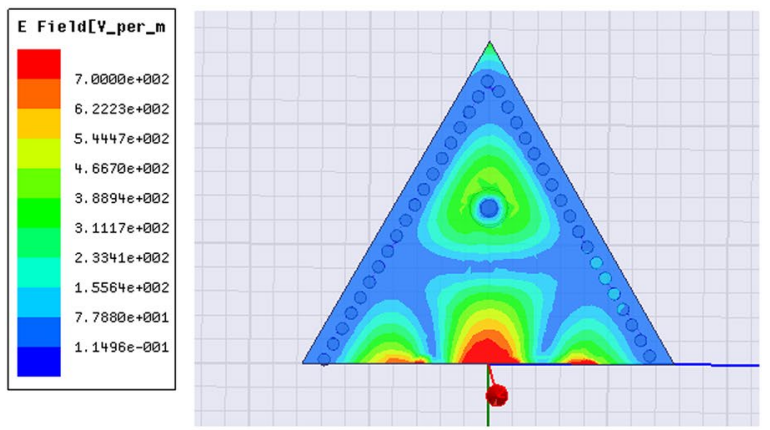

(b)

Fig. 8 Electric field distribution of a rectangular box-shaped DR at $9.34 \mathrm{GHz}$ and $\mathbf{b}$ SIW antenna at $9.68 \mathrm{GHz}$

$5.28 \mathrm{GHz}$ and $9.57 \mathrm{GHz}$ respectively. The antenna gain is measured by gain transfer method using a Krytar $9000 \mathrm{~B}$ power meter, a Hittite HMC-T2100 microwave signal generator $(10 \mathrm{MHz}-20 \mathrm{GHz})$ and a standard gain Horn antenna. For the E-plane, the cut plane is at $\phi=0^{\circ}$ and for the H-plane it is for $\phi=90^{\circ}$. From Fig. $9 a, b$ it is observed that $\mathrm{E}$-plane simulated co-pol values and $\mathrm{H}$-plane gain are $5.7 \mathrm{dBi}$ and $5 \mathrm{dBi}$ respectively with their corresponding values obtained experimentally are $5.6 \mathrm{dBi}$ and $4.9 \mathrm{dBi}$ for the $\mathrm{TE}_{101}$ mode. Similarly, a close look into Fig. 9c, d reveals that for $\mathrm{TE}_{202}$ mode, the simulated and experimental E-plane co-polarized gains are $7.4 \mathrm{dBi}$ and $6.9 \mathrm{dBi}$ 


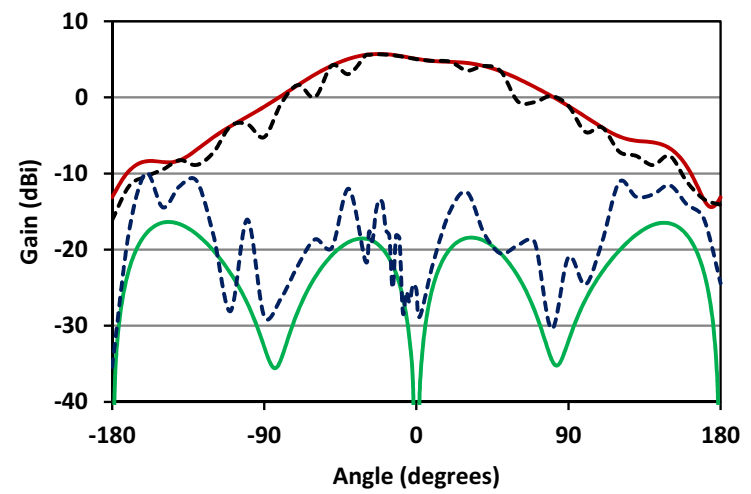

(a)

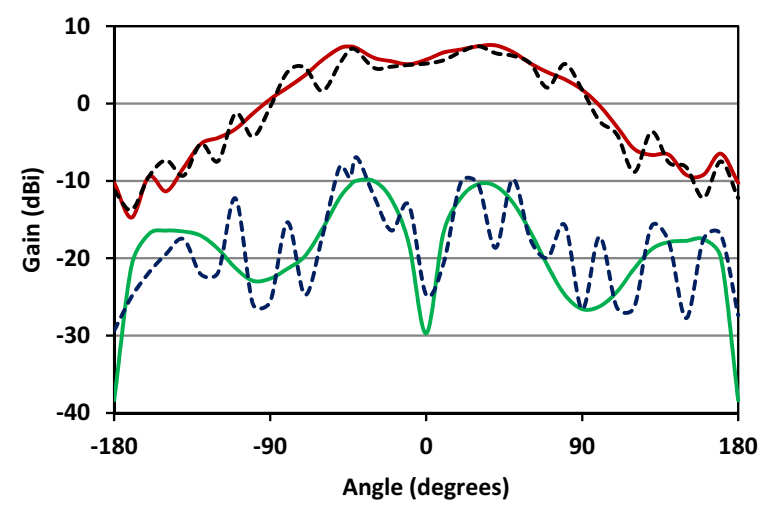

(c)

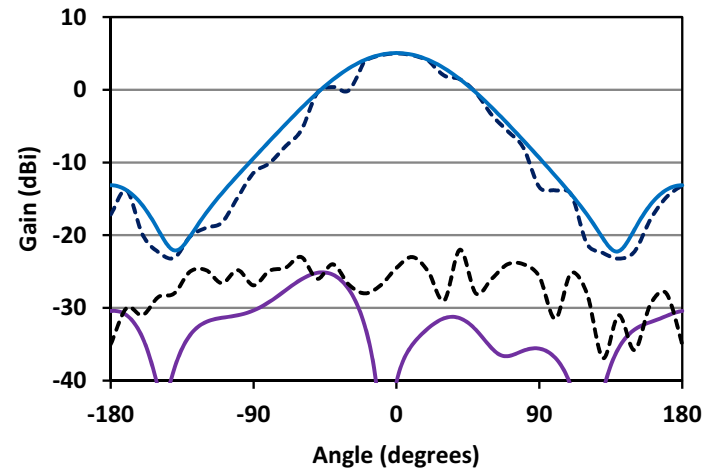

(b)

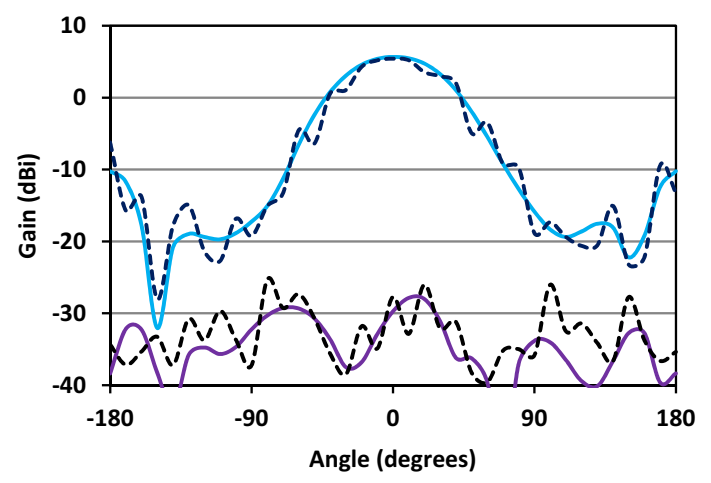

(d)

Fig. 9 Simulated and experimentally obtained gain pattern of the HMSIW based triangular antenna-TE 101 mode at 5.28 GHz-a E-plane radiation pattern and $\mathbf{b} \mathrm{H}$-plane radiation pattern, $\mathrm{TE}_{202}$ mode at $9.5 \mathrm{GHz}-\mathbf{c}$ E-plane radiation pattern and $\mathbf{d} \mathrm{H}$-plane radiation pattern

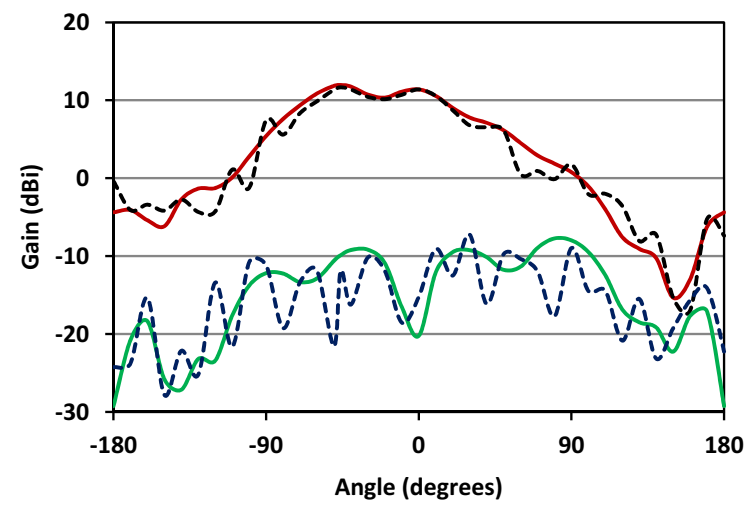

(a)

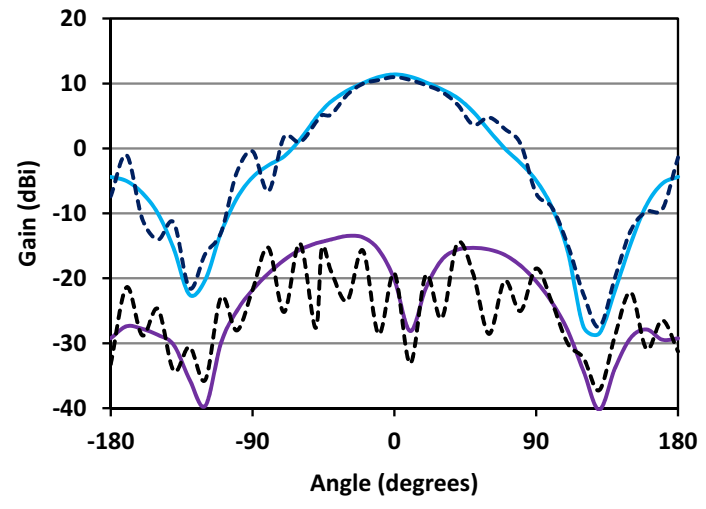

(b)

Fig. 10 Simulated and experimental radiation pattern of the HMSIW triangular antenna with rectangular box-shaped DR for TE 202 mode $(9.5 \mathrm{GHz})$ - $\mathbf{a}$ E-plane radiation pattern and $\mathbf{b}$ H-plane radiation pattern

respectively while the same for $\mathrm{H}$-plane are $5.6 \mathrm{dBi}$ and $5.4 \mathrm{dBi}$ respectively. In both the modes, the cross polarization values are far behind their co-polarized level by $30-40 \mathrm{dBi}$.
Figure 10 reveals that for the antenna-DR composite structure, the E-plane simulated co-polarized gain is $12 \mathrm{dBi}$ with its experimental result counterpart being $11.6 \mathrm{dBi}$. For the H-plane, the simulated and experimentally obtained 


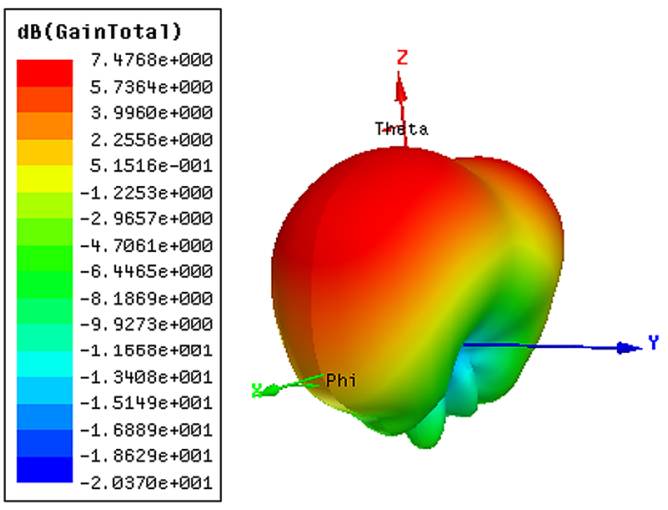

(a)
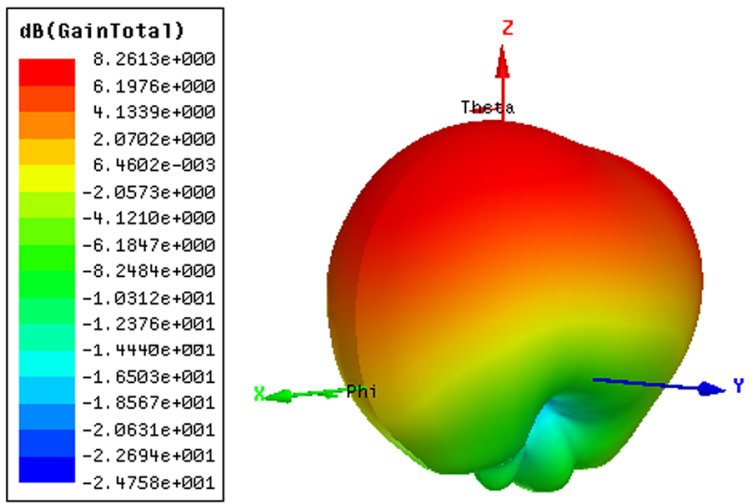

(b)

Fig. $113 \mathrm{D}$ radiation pattern of the HMSIW triangular antenna a without $\mathrm{DR}$ and $\mathbf{b}$ with rectangular box-shaped $\mathrm{DR}$ for $\mathrm{TE}_{202}$ mode (9.5 GHz)

co-polarized gains are $11.4 \mathrm{dBi}$ and $10.9 \mathrm{dBi}$ respectively. For the E-plane, the cut plane is at $\phi=0^{\circ}$ and for the $\mathrm{H}$-plane it is for $\phi=90^{\circ}$. For this antenna supported with DR, the crosspolarized level is approximately $20-30 \mathrm{dBi}$ below its co-polarized value. Figure 11 depicts the 3D radiation pattern of the HMSIW triangular antenna with and without the rectangular box-shaped DR structure. With DR loading, the radiation coming out from the HMSIW equilateral triangular antenna finds a higher permittivity region; hence the beam converges and enhancement of antenna gain occurs. Thus for this antenna an improvement in gain of $4.6 \mathrm{dBi}$ is observed.

\section{Conclusion}

A HMSIW equilateral triangular antenna is taken which resonates at $5.28 \mathrm{GHz}$ at the fundamental $\mathrm{TE}_{101}$ mode and at $9.57 \mathrm{GHz}$ at the next higher $\mathrm{TE}_{202}$ mode. The antenna produces a gain of $5.7 \mathrm{dBi}$ and $7.4 \mathrm{dBi}$ for the two respective modes. This antenna when supported with a rectangular box-shaped dielectric resonator over its PMC wall resonates at the higher $\mathrm{TE}_{202}$ mode at $9.5 \mathrm{GHz}$ producing a gain of $12 \mathrm{dBi}$. Thus an overall enhancement of $4.6 \mathrm{dBi}$ gain is observed with the antenna-DR composite structure. This super high frequency $X$-band high gain antenna would find applications in weather radar systems, in radio location for land, airborne and naval radars and commercial wireless LAN. Thus the proposed antenna would be surely beneficial to be used as a good sensing element for a plethora of X-band microwave imaging and radar applications and lead to further research by researchers worldwide.

\section{Compliance with ethical standards}

Conflict of interest On behalf of all authors, the corresponding author states that there is no conflict of interest.

\section{References}

1. Deslandes D, Wu K (2003) Single-substrate integration technique of planar circuits and waveguide filters. IEEE Trans Microw Theory Tech 51:593-596

2. Xu F, Wu K (2005) Guided-wave and leakage characteristics of substrate integrated waveguide. IEEE Trans Microw Theory Tech 53:66-73

3. Deslandes D, Wu K (2006) Accurate modeling, wave mechanisms and design considerations of a substrate integrated waveguide. IEEE Trans Microw Theory Tech 54:2516-2526

4. Lianlin L, Wenji Z, Fang L (2010) Derivation and discussion of the SAR migration algorithm within inverse scattering problem: theoretical analysis. IEEE Trans Geosci Remote Sens 48(1):415-422

5. Kwon S, Lee S (2016) Recent advances in microwave imaging for breast cancer detection. J. Biomed Imaging, Int

6. Wang Z, Lim EG, Tang Y, Leach M (2014) Medical applications of microwave imaging. Sci World J 2014:147016

7. Smith D, Elsdon M, Leach M, Fernando M, Foti SJ (2006) 3D microwave imaging for medical and security applications. In: IEEE international RF and microwave conference (RFM-2006), pp 233-237

8. Banerjee S, Rana B, Parui SK, Chatterjee S, Dey N (2017) HMSIW based miniaturized sensing antennas for S- and C-band applications. IEEE Sens Lett 1(1):1-4

9. Biswarup R, Parui SK (2017) Microstrip line fed wideband circularly-polarized dielectric resonator antenna array for microwave image sensing. IEEE Sens Lett 1(3):3500604

10. Lai Q, Fumeaux Ch, Hong W, Vahldieck R (2009) Characterization of the propagation properties of the half-mode substrate integrated waveguide. IEEE Trans Microw Theory Tech 57:1996-2004

11. Xu J, Hong W, Tang $\mathrm{H}$, Kuai Z, Wu K (2008) Half-mode substrate integrated waveguide (HMSIW) leaky-wave antenna for millimeter wave applications. IEEE Antennas Wirel Propag Lett 7:85-88

12. Lai QH, Hong W, Kuai ZQ, Zhang YS, Wu K (2009) Half-mode substrate integrated waveguide transverse slot array antennas. IEEE Trans Antennas Propag 57:1064-1072

13. Zelinski GM, Thiele GA, Hastriter ML, Havrilla MJ, Terzouli AJ (2007) Half width leaky wave antennas. IET Microw Antennas Propag 1:341-348 
14. Martinez-Ros AJ, Gomez-Tornero JL, Goussetis G (2012) Planar leaky-wave antenna with flexible control of the complex propagation constant. IEEE Trans Antennas Propag 60:1625-1630

15. Banerjee S, Rana B, Parui SK (2016) Substrate integrated waveguide based triangular antenna arrays. Microw Opt Technol Lett 58(3):675-679

16. Banerjee S, Rana B, Parui SK (2016) SIW based compact and dualband equilateral triangular antennas. J Electromagn Waves Appl (Taylor \& Francis) 30(5):637-650

17. Petosa A, Ittipiboon A (2010) Dielectric resonator antenna: a historical review and the current state of the art. IEEE Antennas Propag Mag 52:91-116

18. Tadjalii A, Sebak AR, Denidni TA (2006) Resonance frequencies and far field patterns of elliptical dielectric resonator antenna: analytical approach. Prog Electromagn Res 64:81-98
19. Song Y, Sebak AR (2006) Radiation pattern of aperture coupled prolate hemispheroidal dielectric resonator antenna. Prog Electromagn Res 58:115-133

20. Cheng J, Rui L, Alphones A, Bao X (2013) Quarter-mode substrate integrated waveguide and its application to antennas design. IEEE Trans Antennas Propag 61:2921-2928

21. Sauer T (2006) Numerical analysis. Pearson Educ, Upper Saddle River

22. Pozar DM (2005) Microwave engineering, 3rd edn. Wiley, New York

Publisher's Note Springer Nature remains neutral with regard to jurisdictional claims in published maps and institutional affiliations. 